\title{
Possible résurgence du virus respiratoire syncytial au Canada
}

\author{
Pascal M. Lavoie MDCM PhD, Frederic Reicherz MD, Alfonso Solimano MD, Joanne M. Langley MD MSc
}

Citation : CMAJ 2021 July 26;193:E1140-1. doi : 10.1503/cmaj.210919-f

Voir la version anglaise de l'article ici : www.cmaj.ca/lookup/doi/10.1503/cmaj.210919

$\mathbf{L}$

'Australie, et plus récemment les États-Unis, ont observé une résurgence des cas d'infection au virus respiratoire syncytial (VRS), alors que les mesures de distanciation physique associées à la pandémie de COVID-19 sont peu à peu levées. Nous soutenons que le Canada devrait anticiper une résurgence comparable des virus respiratoires saisonniers à l'été 2021. Il est essentiel de poursuivre la surveillance des maladies respiratoires afin d'établir les programmes de prévention contre le VRS et de contribuer à la protection des patients vulnérables. Cela pourrait comprendre l'administration hors saison d'un traitement à base d'anticorps monoclonaux contre le VRS chez les enfants à haut risque d'infection, ce qui contrasterait nettement avec la pratique courante.

Avant la pandémie de COVID-19, les infections des voies respiratoires inférieures entraînaient le décès d'environ 2,7 millions de personnes annuellement à l'échelle de la planète, se plaçant au quatrième rang des causes spécifiques de mortalité ${ }^{1}$. Le virus respiratoire syncytial et la grippe sont les principales causes d'infection des voies respiratoires inférieures chez les jeunes enfants. Chez les enfants de moins de 2 ans, le VRS se présente sous la forme d'une bronchiolite et chez les nourrissons, sous la forme de symptômes non spécifiques comme l'apnée et un apport oral réduit. II n'existe pas de vaccin contre le virus respiratoire syncytial et aucune intervention n'est en place au niveau populationnel, mis à part les mesures d'hygiène de base. Au Canada, les enfants à haut risque d'infection de moins de 2 ans notamment les nourrissons prématurés et ceux présentant une forme grave de maladie pulmonaire chronique ou de maladie cardiaque congénitale - reçoivent présentement un traitement mensuel à base d'anticorps monoclonaux pour neutraliser le VRS pendant sa saison de prévalence (typiquement, de la fin de l'automne au début du printemps, au Canada) ${ }^{2}$, ce qui réduit le risque d'hospitalisations associées aux infections des voies respiratoires inférieures par plus de la moitié. Cela se produit sur une base annuelle à date fixe selon l'épidémiologie antérieure, ou sur une base variable selon la surveillance active.

Depuis le début de la pandémie, plusieurs pays ont observé une quasi totale disparition des cas de virus respiratoire syncytial

\section{Points clés}

- L'Australie, et plus récemment les États-Unis, ont observé une résurgence des cas d'infection au virus respiratoire syncytial (VRS) en parallèle avec une réduction des mesures de distanciation physique associées à la pandémie.

- Le Canada devrait aussi anticiper une résurgence comparable et devrait surveiller la maladie respiratoire afin d'être en mesure de répondre rapidement avec de fortes mesures de prévention contre le virus respiratoire syncytial.

- Pendant plusieurs mois, le Canada n'a virtuellement observé aucun cas d'infection au VRS, ce qui pourrait signifier que les femmes enceintes et les nourrissons y ont été moins exposés et de ce fait, les niveaux d'immunité pédiatrique pourraient être bas.

- Une résurgence des cas à l'été 2021 pourrait grever les ressources dans les unités de soins intensifs pédiatriques partout au Canada.

- Les programmes de prévention du virus respiratoire syncytial devraient se préparer à administrer une immunoprophylaxie hors saison aux nourrissons présentant les plus hauts risques d'infection.

et de grippe ${ }^{3,4}$. Au Canada, 339627 tests de dépistage du VRS ont été déclarés au Centre de l'immunisation et des maladies respiratoires infectieuses. De ces tests, 239 étaient positifs entre le 29 août 2020 et le 8 mai $2021^{5}$. En comparaison, on avait déclaré 412861 tests de dépistage du VRS au cours d'une période comparable l'année précédente (du 25 août 2019 au 2 mai 2020), parmi lesquels 18860 étaient positifs. En réponse à l'apparente disparition du virus respiratoire syncytial durant la pandémie, plusieurs programmes canadiens de prévention du VRS ont fait l'objet d'un moratoire, ont été écourtés et ont même suspendu l'administration saisonnière d'anticorps monoclonaux afin d'éviter des consultations médicales inutiles ${ }^{6}$. Cependant, on a récemment signalé une résurgence intersaisonnière de cas du VRS dans l'hémisphère Sud dans les milieux où la circulation du SRASCoV-2 était en diminution et où les mesures de distanciation physique ont été relâchées ${ }^{7,8}$. Les Centers for Disease Control and Prevention (CDC) des États-Unis ont aussi émis une alerte sanitaire le 
10 juin 2021 au sujet d'une augmentation intersaisonnière de l'activité du virus respiratoire syncytial dans le sud des États-Unis depuis le mois de mars 2021 (https://emergency.cdc.gov/ han/2021/han00443.asp - en anglais seulement). La résurgence hors saison des virus respiratoires saisonniers présente possiblement une menace pour les nourrissons vulnérables en ce moment.

La résurgence des cas de VRS en Australie et aux États-Unis souligne l'importance de l'immunité de la population dans le contrôle des épidémies de virus respiratoire syncytial après les pics d'exposition. Des études ont documenté les variations cycliques des niveaux d'anticorps maternels, avec une augmentation au cours de la saison hivernale du VRS, suivie d'un déclin au cours des mois estivaux 9 . Au cours des saisons du virus respiratoire syncytial, les personnes adultes de moins de 65 ans qui sont les plus affectées restent asymptomatiques ou développent une forme légère de la maladie grâce à leur exposition saisonnière au virus depuis leur enfance ainsi qu'à l'immunité des lymphocytes $B$ et $T$ mémoires. Par opposition, les nourrissons qui ne possèdent pas d'immunité dépendent du transfert passif des anticorps maternels pour les protéger à la naissance, mais la protection de ces anticorps s'amenuise sur une période de 6 mois. Le dépistage asymptomatique des ménages indique que la transmission pourrait s'être poursuivie sans être détectée pendant la pandémie de COVID-1910, mais il est plus probable qu'au cours de la pandémie, les personnes enceintes étaient moins susceptibles d'être exposées au VRS et ainsi, avaient moins de chance d'amplifier la production de leurs anticorps contre le VRS aux niveaux observés pendant l'hiver. Cela soulève la possibilité que les nourrissons soient moins bien protégés qu'à l'habitude et qu'ils puissent être plus malades s'ils sont infectés cet été.

En Australie, au cours de la récente résurgence du virus respiratoire syncytial, l'âge médian des enfants chez qui le dépistage du VRS s'est avéré positif a augmenté à 18,4 mois, ce qui dépassait la limite supérieure observée entre 2012 et 2019 (7,3$12,5$ mois; $p<0,001)^{7}$. Cela suggère que les nourrissons qui n'ont pas été exposés au VRS au cours de leur première année de vie n'ont pas développé une immunité suffisante, de sorte qu'ils sont demeurés vulnérables au virus au cours de leur deuxième année. En Australie, les intensivistes pédiatriques ont signalé une augmentation des cas graves et atypiques d'infections au virus respiratoire syncytial dans les 4 premiers mois de 2021 (fin de l'été, début de l'automne dans les mois suivant le confinement) chez des enfants plus âgés nécessitant une ventilation mécanique (Trevor Duke, Hôpital pédiatrique Royal Children, Melbourne, Australie - communication privée). Si un phénomène comparable survenait au Canada, le fardeau de la maladie pourrait grever les ressources dans les unités de soins intensifs pédiatriques au cours de l'été 2021. Bien que nous ayons été incapables de trouver des données publiées portant sur des modifications aux réponses de santé publique aux résurgences hors saison des cas de VRS et de grippe, nous savons que certains États australiens ont commencé des traitements aux anticorps monoclonaux à l'avance et ont décidé de prolonger l'immunoprophylaxie contre le virus respiratoire syncytial jusqu'à ce que le nombre de cas se résorbe.
En prévision d'une possible résurgence du VRS au Canada, nous suggérons l'approche suivante. Premièrement, alors que les mesures de distanciation physique liées à la pandémie sont relâchées, les fournisseurs de soins de santé devraient continuer à mettre les mesures d'hygiène de base de l'avant, comme le lavage des mains. Nous soutenons les efforts continus afin d'empêcher que les nourrissons vulnérables n'entrent en contact avec des personnes atteintes de maladies respiratoires. D'autres mesures de protection, comme l'allaitement maternel lorsque cela est possible et l'évitement d'une exposition à la fumée secondaire, devraient se poursuivre et être accentuées. Deuxièmement, les tests de contrôle du VRS devraient se poursuivre selon les indications habituelles afin de fournir des données de surveillance. Troisièmement, les unités de soins intensifs pédiatriques devraient anticiper une augmentation des cas graves de virus respiratoire syncytial, comme signalés en Australie$^{7}$. Les programmes de prévention du VRS devraient se préparer à administrer une immunoprophylaxie hors saison aux nourrissons présentant les plus hauts risques d'infection si les cas augmentent aux niveaux correspondant au déclenchement habituel de la saison automnale.

Au Canada, les programmes de prévention du VRS n'ont pas encore défini de façon prospective le nombre de cas d'infection au VRS qui constituerait une résurgence, mais il semble raisonnable de croire que même quelques cas devraient provoquer des échanges entre les parties prenantes, surtout en prenant en considération que pendant plusieurs mois nous n'avons observé aucun cas pendant plusieurs semaines dans un grand nombre de provinces et de territoires. Néanmoins, il convient de mentionner que la rentabilité du traitement aux anticorps monoclonaux hors saison contre le VRS n'est pas déterminée dans le présent contexte. Nous n'anticipons pas de pénuries de médicaments, mais de nouvelles ententes doivent être coordonnées pour l'envoi et l'entreposage de palivizumab plus tôt qu'à l'habitude, en préparation d'une résurgence du virus respiratoire syncytial. Finalement, des études sont nécessaires afin de documenter les répercussions de la pandémie sur l'occurrence cyclique des virus saisonniers et pour comprendre pourquoi le VRS et la grippe sont disparus au cours de la dernière année, alors que le SRAS-CoV-2 et les rhinovirus ont continué à circuler ${ }^{11}$.

\section{Références}

1. GBD 2015 Mortality and Causes of Death Collaborators. Global, regional, and national life expectancy, all-cause mortality, and cause-specific mortality for 249 causes of death, 1980-2015: a systematic analysis for the Global Burden of Disease Study 2015. Lancet 2016;388:1459-544.

2. Obando-Pacheco P, Justicia-Grande AJ, Rivero-Calle I, et al. Respiratory syncytial virus seasonality: a global overview. J Infect Dis 2018;217:1356-64.

3. Yeoh DK, Foley DA, Minney-Smith CA, et al. The impact of COVID-19 public health measures on detections of influenza and respiratory syncytial virus in children during the 2020 Australian winter. Clin Infect Dis 2020;72:2199-2202.

4. Rodgers L, Sheppard M, Smith A, et al. Changes in seasonal respiratory illnesses in the United States during the COVID-19 pandemic. Clin Infect Dis 2021 Apr 29 [cyberpublication avant impression]; ciab311. doi: 10.1093/cid/ciab311.

5. Respiratory virus detections in Canada. Ottawa: Public Health Agency of Canada; 2021. Accessible ici : https://www.canada.ca/en/public-health/services/surveillance /respiratory-virus-detections-canada.html (consulté le 12 mai 2021). 
6. Taylor RS. Respiratory syncytial virus and palivizumab prophylaxis in the COVID-19 era [lettre]. CMAJ 2021;193:E523.

7. Foley DA, Yeoh DK, Minney-Smith CA, et al. The interseasonal resurgence of respiratory syncytial virus in Australian children following the reduction of coronavirus disease 2019-related public health measures. Clin Infect Dis 2021 Feb 17 [cyberpublication avant impression];ciaa1906. doi: 10.1093/cid/ciaa1906.

8. McNab S, Ha Do LA, Clifford V, et al. Changing epidemiology of respiratory syncytial virus in Australia - delayed re-emergence in Victoria compared to WA/ NSW after prolonged lock-down for COVID-19. Clin Infect Dis 2021 Mar 18 [cyberpublication avant impression];ciab240. doi: 10.1093/cid/ciab240.

9. Stensballe LG, Ravn H, Kristensen K, et al. Seasonal variation of maternally derived respiratory syncytial virus antibodies and association with infant hospitalizations for respiratory syncytial virus. J Pediatr 2009;154:296-8.

10. Emanuels A, Heimonen J, O'Hanlon J, et al. Remote household observation for non-influenza respiratory viral illness. Clin Infect Dis 2020 Nov 17;ciaa1719 [cyberpublication avant impression]. doi: 10.1093/cid/ciaa1719.

11. Baker RE, Park SW, Yang W, et al. The impact of COVID-19 nonpharmaceutical interventions on the future dynamics of endemic infections. Proc Natl Acad Sci U S A 2020;117:30547-53.

Intérêts concurrents : Pascal Lavoie et Alfonso Solimano siègent au comité exécutif, et Alfonso Solimano est le directeur médical, du Programme d'immunoprophylaxie pour la prévention des infections par le virus respiratoire syncytial (VRS) de la Colombie-Britannique, un programme financé par l'État sous la responsabilité de la Régie provinciale des services de santé du ministère de la Santé de la Colombie-Britannique. Le site d'étude de Joanne Langley a réalisé des travaux de recherche sur les vaccins contre le virus respiratoire syncytial en collaboration avec GSK, Janssen et Pfizer. Le soutien financier de ces études provient de l'Université Dalhousie. Aucun autre intérêt concurrent n'a été déclaré.

Cet article a été révisé par des pairs.

Affiliations : Division de néonatalogie, Département de pédiatrie (Lavoie, Solimano), Université de la Colombie-Britannique, Institut de recherche de l'Hôpital pour enfants de la Colombie-Britannique (Lavoie, Reicherz), Vancouver, C.-B.; Centre canadien de vaccinologie, Université Dalhousie (Langley), Centre de soins de santé IWK et Régie de la santé de la Nouvelle-Écosse, Halifax, N.-É.

Collaborateurs : Tous les auteurs ont contribué à l'élaboration et à conception de l'étude ainsi qu'à l'ébauche du manuscrit, ont révisé de façon critique son contenu intellectuel important, ont donné leur approbation finale pour la version destinée à être publiée et endossent l'entière responsabilité de tous les aspects du travail.

Propriété intellectuelle du contenu : Il s'agit d'un article en libre accès distribué conformément aux modalités de la licence Creative Commons Attribution (CC BY-NC-ND 4.0), qui permet l'utilisation, la diffusion et la reproduction de tout médium à la condition que la publication originale soit adéquatement citée, que l'utilisation se fasse à des fins non commerciales (c.-à-d., recherche ou éducation) et qu'aucune modification ni adaptation n'y soit apportée. Voir : https://creativecommons.org/licenses/by-nc-nd/4.0/deed.fr.

Remerciements : Les auteurs remercient les $D^{\text {rs }}$ Manish Sadarangani, David Goldfarb et Joan Robinson pour les discussions suivies et judicieuses sur cet enjeu et pour leur avis d'experts sur ce commentaire; ainsi que Cheryl Christopherson pour son examen des programmes provinciaux de prévention du VRS en pratique durant la pandémie.

Correspondance : Pascal Lavoie, plavoie@cw.bc.ca 